\title{
J-3-Gaussian Beam Distortion Caused by Saturable Gain or Loss
}

\author{
WILLIAM B. BRIDGES, MEMBER, IEEE
}

\begin{abstract}
Results are given for the beam distortion suffered by low order Gaussian-Laguerre modes propagating through a saturable laser amplifier. Both uniform and Bessel small-signal gain distributions are considered. Beam narrowing caused by saturable loss is also treated.
\end{abstract}

\section{INTRODUCTION}

T IHIS paper is concerned with a particular type of spatial distortion that can occur as an optical signal propagates through a laser amplifier. Although spatial distortion ("lensing," a transverse redistribution of energy, or some other distortion) can arise as the result of any of a number of physical processes, we shall consider here the effects of only one physical process, gain saturation. The phenomenon considered in this paper may be stated simply: a signal with a transverse distribution of intensity propagating through a saturable medium suffers distortion because of the saturationinduced change in the distribution of gain. The weaker portions of the signal are amplified relatively more than the stronger portions because they saturate the medium to a lesser degree. For Gaussian beams the distortion results in a general broadening of the beam for a medium with saturable gain, and a narrowing for a medium with saturable loss. For the higher order Gaussian modes, the nulls (dark rings) become narrower and more "filled in,"

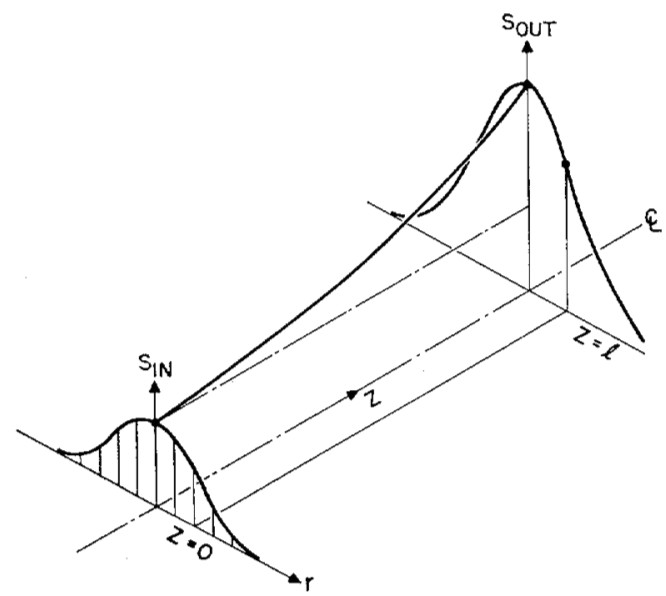

Fig. 1. Schematic representation of the coordinate system used in the model. An input distribution $S_{\text {in }}$ is specified at $z=0$, and allowed to propagate parallel to the optical axis. The output distribution $S_{\text {out }}$ at $z=1$ is computed by integrating the saturation equation (1) or (2) along the path.

Manuscript received June 13, 1968. This research was supported in part by the Office of Naval Research. This paper was presented at the 1968 International Quantum Electronics Conference, Miami, Fla.

The author is with Hughes Research Laboratories, Malibu, Calif. while the off-axis maxima become relatively more intense' ultimately approaching the on-axis intensity.

There are other physical processes that also produce beam distortion, notably those that modify the medium index of refraction and distort the wavefronts of the propagating beam. These effects may be greater or smaller in their contribution to the net beam shape than the simple effect considered here. However, since it is the intent of this present calculation to show only the effect of the physical process described above, we explicitly omit these effects and consider a very simple mathematical model. Fig. 1 illustrates the model used for calculation. Because axisymmetric ${ }^{1}$ modes are of greatest interest (especially the TEM $_{00}$ mode), only the coordinates $(r, z)$ need be considered. The radial intensity distribution $S(r, z)$ is specified at the input plane $z=0$ as $S_{\text {in }}(r, 0)$. The output intensity distribution $S_{\text {out }}(r, l)$ is then computed at $z=l$ from the equation

$$
\frac{d S}{d z}=\frac{g_{o} S}{1+\frac{S}{S_{o}}}
$$

for the case of pure homogeneous interaction, or

$$
\frac{d S}{d z}=\frac{g_{0} S}{\left(1+\frac{S}{S_{o}}\right)^{1 / 2}}
$$

for the case of a pure inhomogeneous interaction [2].

The quantities $g_{o}$ and $S_{o}$, respectively, are the smallsignal gain in meter ${ }^{-1}$ and the saturation parameter in $\mathrm{W} / \mathrm{m}^{2}$. Equation (1) or (2) is integrated numerically step by step in the $z$-direction ${ }^{2}$ along a path parallel to the

1 The calculations given here were made for the TEM To , TEM ${ }_{01}^{*}$, and TEM $_{10}$ Gaussian-Laguerre modes. While the TEM $_{00}$ and TEM 10 modes are axisymmetric, the $\mathrm{TEM}_{01}{ }^{*}$ mode is usually described as being an axisymmetric mode composed of two nonaxisymmetric TEM 01 modes superposed in time and space quadrature [1]. The calculation in the present paper was made simply by taking the distribution for the TEM 01 mode with $\phi=0$, which falls along the null line of the quadrature TEM that any input distribution may be used, not just the axisymmetric Gaussian-Laguerre modes. An interesting problem left to the reader is how to find the input distribution that produces the TEM $\mathbf{T E}_{00}$ mode at the output for a given set of conditions.

2 We should point out that for the simple model shown in Fig. 1, (1) and (2) can be solved in closed form and need not be integrated numerically. However, the closed-form solutions give $S$ implicitly rather than explicitly, and must still be evaluated numerically to find $S_{\text {out }} / S_{\text {in. }}$. The forward integration in $z$ proceeds almost as rapidly as the evaluation, even on small computers, and also allows an easy extension of the model to situations that do not have closed form solutions (e.g., the situation that occurs when a first order correction is added for diffraction as discussed later or for spatial cross-relaxation). 
axis for several different values of $r$, usually $0 \leq r \leq 2 w_{o}$, where $w_{0}$ is Gaussian beam parameter at the beam waist. (For a collimated TEM on $_{0}$ mode, it is the radius at which the beam intensity falls to $1 / e^{2}$.) When significant signal intensity is found to fall outside $r / w_{o}=2$, the calculation is repeated for larger values of $r$ until the output signal intensity at still greater radii is small enough to be neglected. The interval between successive values of $r$ was usually taken as $\Delta r / w_{0}=0.1$, although smaller values were used to determine the finer structure in the higher order modes. The four-point Milne formula [3] for the derivative $S^{\prime}$

$$
S_{n}^{\prime}=S_{n-4}^{\prime}+\frac{4}{3} \Delta z\left(2 S_{n-3}^{\prime}-S_{n-2}^{\prime}+2 S_{n-1}^{\prime}\right)
$$

was used for the numerical integration, with $\Delta z / l$ usually taken as 0.01 . Computations were made for a range of small-signal gain and saturation parameters. A convenient way of expressing these parameters is as the total small-signal gain in decibels and a normalized input signal $\left[P_{\mathrm{in}} / S_{0}\left(\pi w_{0}^{2} / 2\right)\right] \mathrm{dB}$, where $P_{\text {in }}$ is the total input signal power in watts. It is easy to show that

$$
P_{\text {in }}=\left(\frac{\pi w_{0}^{2}}{2}\right) \int_{0}^{\infty} S_{\text {in }}(r, 0) 2 \pi r d r
$$

for any Gaussian-Laguerre mode [4]. Thus a normalized input signal value of $0 \mathrm{~dB}$ represents a single flux approximately equal to the saturation flux of the laser medium.

In addition to calculating the radial intensity distribution at the output plane $z=l$, the integral of this distribution was also taken in order to calculate $P_{\text {out }}$ and the true power gain $P_{\text {out }} / P_{\text {in }}$, which, because of the distortion, is no longer equal to $S(0, l) / S(0,0)$. In addition, such quantities as the $3-\mathrm{dB}$ radius, the $1 / e^{2}$ radius, and the radius containing 90 percent of the power were also calculated by simple interpolation from the distribution and its integral. A sample program written in BASIC for the GE 265 time-sharing system is listed in the Appendix. Two properties of the distribution are always preserved in this simple model. Zeros in the input distribution are always continued at the same radius as zeros, and maxima (or minima) in the input always produce maxima (or minima) at the same radius in the output. The only distribution that is transmitted undistorted through an amplifier (according to this model) is the uniform distribution, because it produces a uniformly saturated gain with radius, and then only for the case of uniform small-signal gain, $g_{0} \neq g_{o}(r)$.

With the above description of the mathematical model used it is clear which physical processes have been ignored:

1) Diffraction [4].

2) The radial index variation resulting from an imposed radial variation of gain [5].

3) The radial index variation resulting from the saturation-induced radial variation of gain [6].

4) Beam guiding resulting from a radial gain variation, whether imposed [7], [8] or saturation-induced.

5) All nonlinear beam trapping effects that depend on powers of the electric field higher than 2 (e.g., Kerr effect etc. [9]).
6) The radial index variation induced thermally as a result of energy deposited by the beam as it passes through $[10]$.

It is fair to ask, having omitted all these processes, under what conditions, if any, the results of the computation are valid; that is, under what conditions could they be experimentally observed without interference from processes 1) through 6)? Diffraction may be legitimately ignored provided the medium length $l$ is $\ll w_{o}^{2} / \lambda$. This condition is usually satisfied in high gain opticallypumped solid-state laser amplifiers such as ruby. It may or may not be satisfied in the high gain infrared gas laser amplifiers using $\mathrm{CO}_{2}$ or $\mathrm{Xe}$. For example, a 1-cm-diameter beam in a $10.6-\mu$ laser amplifier has $w_{o}^{2} / \lambda \approx 10 m$,while considerably longer path lengths have been used with beams approximately this size [11], [12]. A first order correction to the present calculation could be made for cases where $w_{o}^{2} / \lambda$ is not much larger than unity by assuming the radius of the input beam expands according to the Gaussian beam formula [4]

$$
r(z)=r(0)\left[1+\left(\frac{\lambda z}{\pi w_{0}^{2}}\right)^{2}\right]^{1 / 2}
$$

by taking the integration along such curved paths and reducing the flux density $S$ by the appropriate area expansion ratio. Of course, (5) is valid only for Gaussian beams, and the beam is no longer Gaussian once distortion occurs. The complete Fresnel integral formulation would then have to be used (in which case the phasefront-distorting processes might as well be included also; the model would have become sufficiently complicated by this time). The correction given by (5) was not used in the present calculations.

The lens action caused by the radial variations may be ignored provided the input signal frequency coincides with the center of the amplifier gain curve, and provided the gain curve is symmetrical about its center. The dispersion relation then gives a refractive index equal to 1 , independent of radius. Processes 5) and 6) primarily occur in external media or absorbers rather than in laser amplifiers themselves. We may indeed encounter some difficulty experimentally verifying the simple model for absorbers. Such experimental verification would require the existence of an "easily" saturable material, that is, one that will be well saturated at sufficiently low intensities so that processes 5) and 6) do not occur first.

Process 4), beam guiding, remains the only process that. cannot obviously be eliminated by some choice of experimental parameters. It is difficult to estimate the relative importance of the two effects, since the theory has only been given by Kogelnik [7] for a nonsaturated medium that is uniform in the $z$-direction. The recent experimental confirmation of this theory by Casperson and Yariv [8] for modes in an oscillator was also made under these conditions. It is clear that the present model, by its very nature, produces serious radial variations: in gain, which should give rise to the same phenomena described in [7] and [8]; however, the amount of radial 
variation varies with $z$ and was calculated by ignoring such beam guiding. Clearly, the two models should be combined to give a better picture of beam distortion, but this will again require the solution of a nonlinear nonuniform wave equation, which is beyond the scope of this paper.

It may indeed be impossible to separate out effect (4) when the medium saturates and observe the simple beam distortion predicted by this model. The devices described in [11] and [12] have not yet been sufficiently free from diffraction and wall reflection effects to give unequivocal measurements of the output distribution. Recent observations by Carbone [13] on a smaller $\mathrm{CO}_{2}$ laser amplifier and by Mikaeliane et al. on a ruby laser amplifier [14] are encouraging in this respect however. The latter work also includes measurements of the angular beam divergence that accompanies the apparently saturation-broadened beam distribution, showing a decreased divergence. At least small values of such spatial distortion do not appear to be harmful.

\section{Results of the Calculations}

Fig. 2 shows the change in the radial intensity distribution for a $\mathrm{TEM}_{00}$ beam (dotted line) entering a homogeneously broadened laser amplifier with a radially uniform small-signal gain of $40 \mathrm{~dB}$. The values of the normalized signal parameter $\left[P_{\mathrm{in}} / S_{0}\left(\pi w_{o} / 2\right)^{2}\right]$ are given in decibels for the different solid curves; the arrow shows the direction of increasing input power. The output distribution has been normalized to its value on the amplifier axis to make the distortion more evident. Fig. 2 (a) shows that even for input signals $40 \mathrm{~dB}$ below the saturation flux (that is, a signal that would begin to saturate the medium only near the amplifier exit) there is about a 10 percent expansion of the $e^{-2}$ radius. When the input has increased to a value that begins to saturate the amplifier at its input ( 0 -dB curve), the $e^{-2}$ radius has just about doubled. However, the distribution nearer the axis $\left(r / w_{0}<1\right)$ has begun to shrink slightly. Fig. 2(b) shows the results for a further increase in input signal. (The input distribution (dotted) and the $0-\mathrm{dB}$ curve are repeated for reference.) The $e^{-2}$ radius increases slightly more at $+5 \mathrm{~d}$ B input, but otherwise falls below the 0 - $\mathrm{d} B$ curve. The $20-\mathrm{dB}$ curve (representing an input flux 100 times the saturation flux) very closely approaches the input curve in shape for $r / w_{0}<1$, but still lies significantly above it for $r / w_{0}>1$. Thus, it would seem that the maximum distortion (or expansion) occurs when the input signal is about equal to the saturation flux. For either extreme, $S \ll S_{0}$ or $S \gg S_{o}$, the output resembles the input in shape. These limits could have been deduced directly from (1); for $S \ll S_{o}$, which requires not only $S_{\text {in }} \ll S_{\text {o but }} S_{\text {in }} \exp \left(g_{0} l\right) \ll S_{o}$, (1) reduces to

$$
\frac{d S}{d z}=g_{0} S
$$

with the solution

$$
S_{\text {out }}=S_{\text {in }} \exp \left(g_{o} l\right)
$$

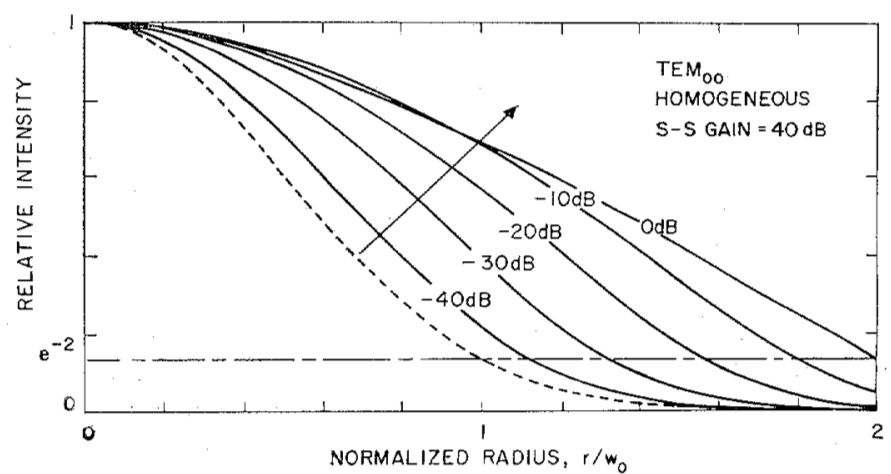

(a)

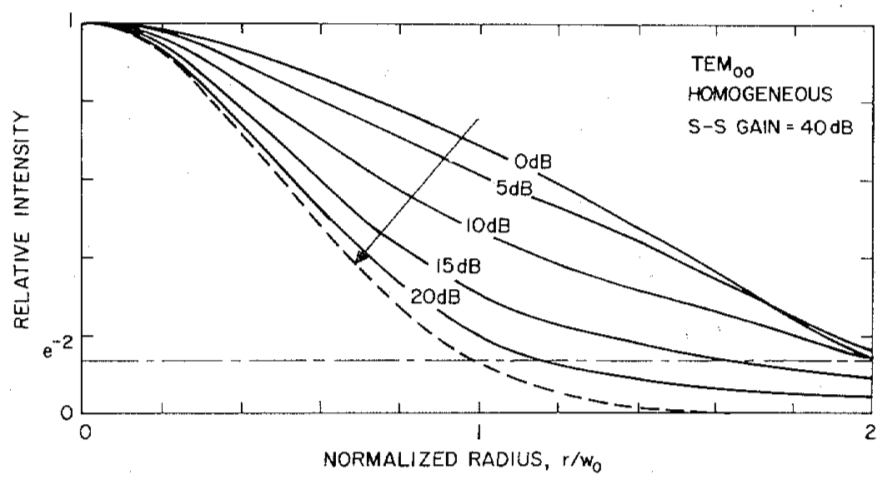

(b)

Fig. 2. The spreading of a $\mathrm{TEM}_{00}$ input beam (shown dashed) due to homogeneous gain saturation. The small-signal gain specified by $g_{o} l$ is $40 \mathrm{~dB}$ and is assumed to be uniform with radius. The normalized input power is given in decibels on each curve. (a) shows the broadening as the normalized input signal intensity is increased from $-40 \mathrm{~dB}$ to $0 \mathrm{~dB}$, while (b) shows the narrowing that occurs upon further increase in signal intensity from $0 \mathrm{~dB}$ to $20 \mathrm{~dB}$.

so that if $g_{0} l$ is independent of $(r, z), S_{\text {out }}$ will have the same distribution as $S_{\text {in }}$. At the other extreme, $S \gg S_{o}$, (1) reduces to

$$
\frac{d S}{d z}=g_{o} S_{o}
$$

with the solution

$$
S_{\text {out }}=S_{\text {in }}+g_{o} l S_{a}
$$

which states that $S_{\text {out }}$ is the same as $S_{\text {in }}$ plus a small additive constant. For the curve in Fig. 2(b) marked "20 dB", $g_{0} l S_{0} \approx 10$ percent of $S_{\text {in }}(r=0)$, so that the additive constant is small, and (9) would predict that the output resembles the input. Another way of stating this is that with signals this large, the amplifier is so highly saturated that it adds very little to the signal passing through and thus cannot distort it.

Fig. 3 shows the behavior of the $e^{-2}$ radius with input signal level for amplifiers with small signal gains of 10 to $80 \mathrm{~dB}$. The curve labeled " $40 \mathrm{~dB}$ " in Fig. 3 is simply a trace of the intersections of the curves in Fig. 2 with the $e^{-2}$ line. All the curves approach an $e^{-2}$ radius of 1 for sufficiently small signals, but for a small-signal gain of $80 \mathrm{~dB}$ the normalized input must be $\ll-80 \mathrm{~dB}$, which is off the scale range shown in Fig. 3 .

Fig. 3 also shows curves for homogeneously saturable loss. These show the contraction of the $e^{-2}$ radius when 


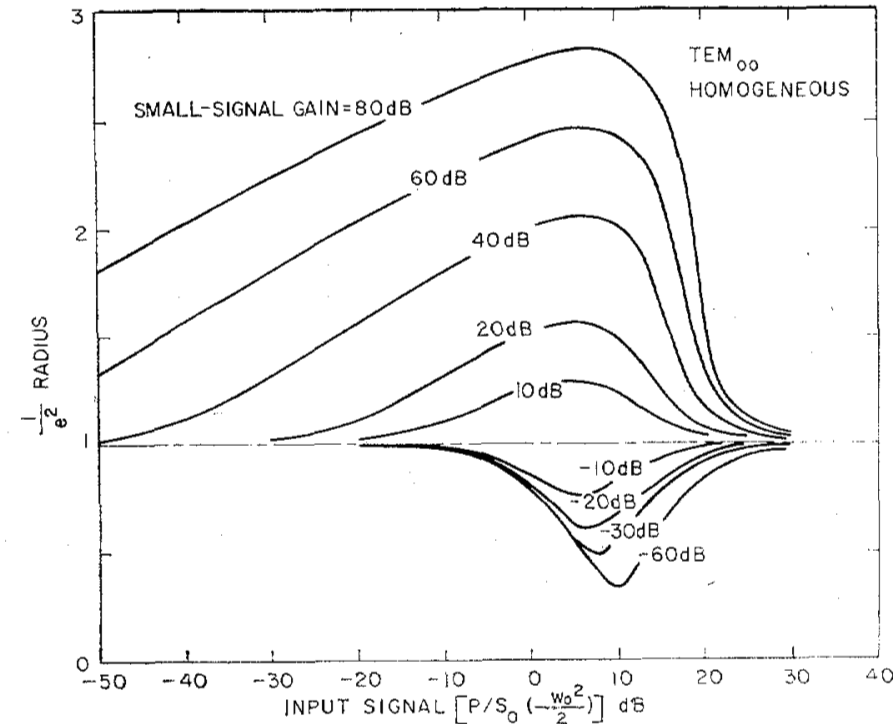

Fig. 3. The $e^{-2}$ radius as a function of the normalized input power in decibels. The small-signal gain values are given on the curves. The results of both gain and loss calculations are shown for a TEM $_{00}$ beam assuming homogeneous interaction.

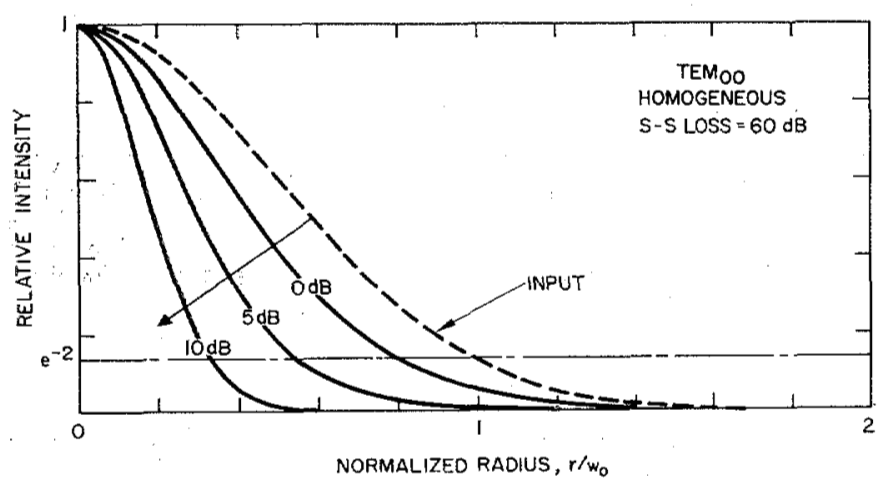

(a)

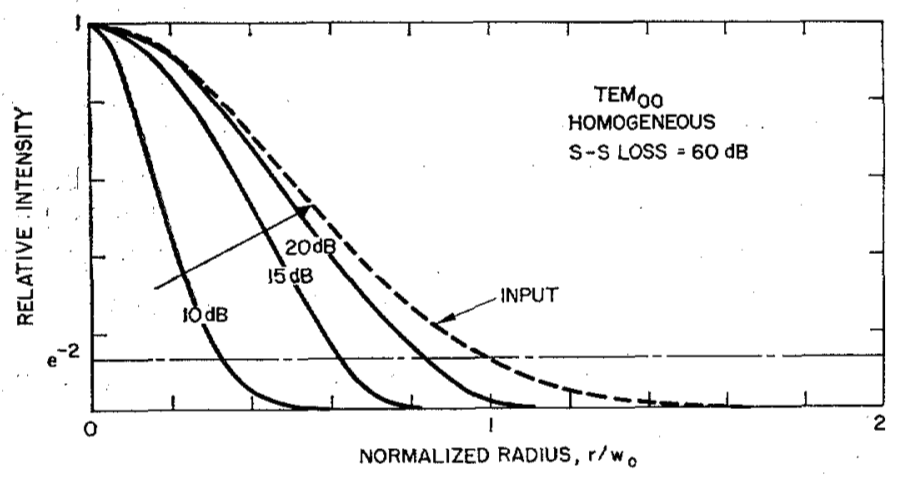

(b)

Fig. 4. Contraction of a TEM 00 input beam (shown dashed) due to homogeneous loss saturation. The small-signal loss is $-60 \mathrm{~dB}$ and is uniform with radius. (a) shows the contraction for input signals of $0 \mathrm{~dB}$ to $10 \mathrm{~dB}$, while (b) shows the reexpansion for input signals, $10 \mathrm{~dB}$ to $20 \mathrm{~dB}$.

signals of the order of the saturation flux are incident. Fig. 4 shows the actual change in output distribution for different signal levels when saturable loss is encountered. In principle, these curves could be computed with exactly the same program by simply inserting negative values of $g_{0}$. In practice, it was necessary to perform the forward

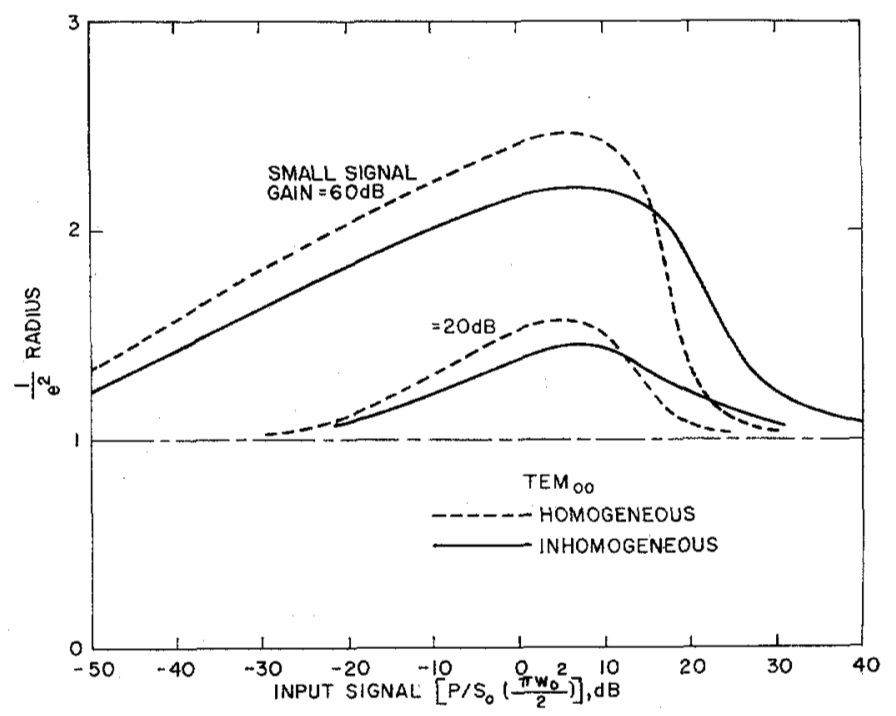

Fig. 5. Comparison of the beam expansion at the $e^{-2}$ radius for homogeneous interaction (dashed curves, repeated from Fig. 3) and inhomogeneous interaction for two values of small-signal gain.

integration with a new variable

$$
S^{(-)} \equiv S \exp \left[-g_{0} l\right]
$$

when negative values of $g_{0}$ were used, otherwise, the finite differences became so small that roundoff error led to inaccurate results for large values of loss.

Fig. 5 compares the position of the $e^{-2}$ radius for homogeneous interaction, (1), and inhomogeneous interaction, (2). The small-signal behavior is quite similar for the two kinds of interaction, as it should be, since both (1) and (2) have (7) as a solution when $S \ll S_{0}$. However, when $S \gg S_{o}$, (2) becomes

$$
\frac{d S}{d z}=g_{0} S_{o}^{1 / 2} S^{1 / 2}
$$

with the solution

$$
S_{\mathrm{out}}=S_{\mathrm{in}}+g_{o} l S_{o}\left(\frac{S_{\mathrm{in}}}{S_{o}}\right)^{1 / 2}+\left(\frac{g_{o} l}{2}\right)^{2} S_{o}
$$

Comparing this with (9) we see that the additive term of (9) is now multiplied by a factor larger than unity, which increases as $\left(S_{\mathrm{in}}\right)^{1 / 2}$, plus another additive constant. Thus the difference between output and input distributions persists to larger values of $S_{\mathrm{in}}$.

Fig. 6 compares the gain saturation curves that would be obtained for two different measurement methods. The solid curves would be obtained by measuring the power in the entire output beam and dividing by the power in the entire input beam. This yields the true power gain of the amplifier. The dashed curves would result if a small detector were used to sample the power on axis at the output and input to determine the ratio, the onaxis gain. The two methods would be equivalent if it were not for the distortion, of course; indeed, the curves fall on top of one another at sufficiently small signal levels, as shown by the 20-dB curves. Experimentally measured gain saturation data are often fit to curves such as these (usually the on-axis curve) in order to determine 


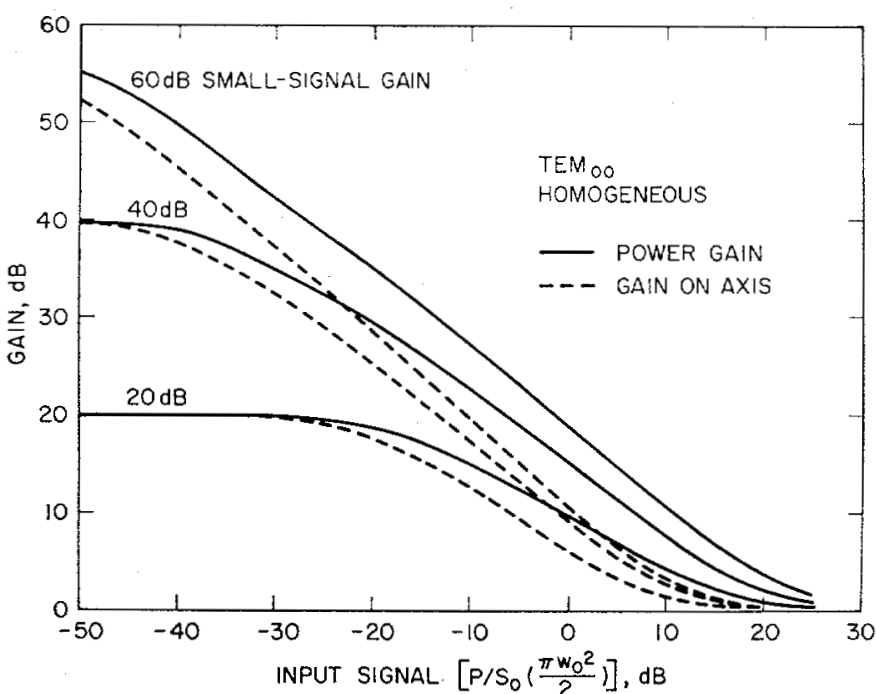

Fig. 6. Gain saturation curves that would be obtained by ratios of total output and input power (solid curves) and on-axis power densities (dashed curves). Results are given for 20-, 40-, and 60-dB small-signal gain and a TEM $_{00}$ mode, homogeneously saturated.

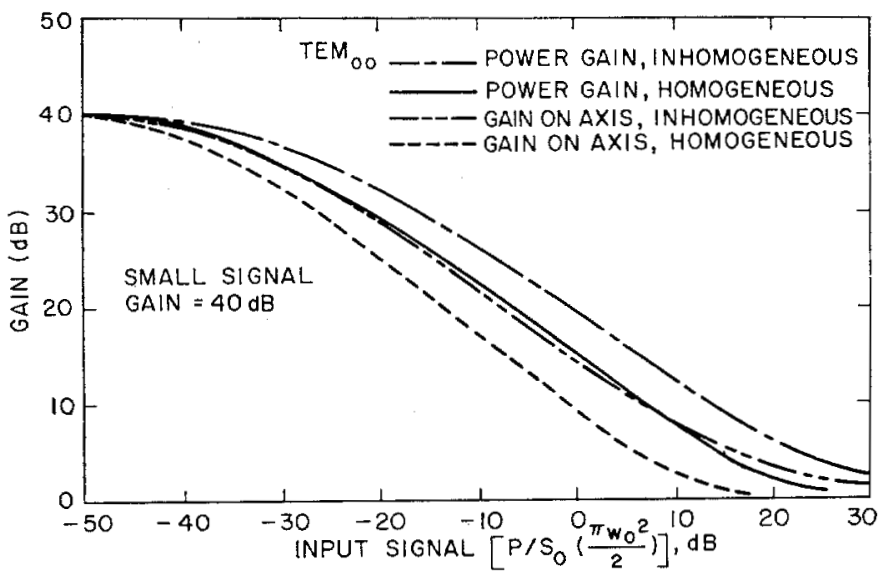

Fig. 7. Gain saturation curves for the four possible combinations of measurement method and type of interaction for $40-\mathrm{dB}$ smallsignal gain.

the saturation parameter $S_{\circ}[11]$, [12], [15], [16]. However, one must be careful to fit the data to the curve appropriate to the method used to take the data. We note that at the 10-dB gain level, the " $20 \mathrm{~dB}$ " curves differ by $5 \mathrm{~dB}$ in horizontal separation, which could result in approximately a 5 -dB error in the value obtained for $S_{0}$ unless the data were fitted to the correct curve. For the "60 dB" curve, the error could be $10 \mathrm{~dB}$ or a factor of 10 . This may serve to explain some of the divergence of results obtained for $S_{o}$ in [11], [12], [15], and [16]. In all of the preceding work it is assumed that no aperturing of the beam takes place by the amplifier tube walls or any other obstacle, so that a beam is free to expand by a factor of 2 (for example) in the 40-dB small-signal gain case; the most common experimental problem encountered in determining a value of $S_{o}$ by this means is to make certain that all the input signal is transmitted through the amplifier without aperturing, so that the entire output can be measured. For this reason we must also ascribe some

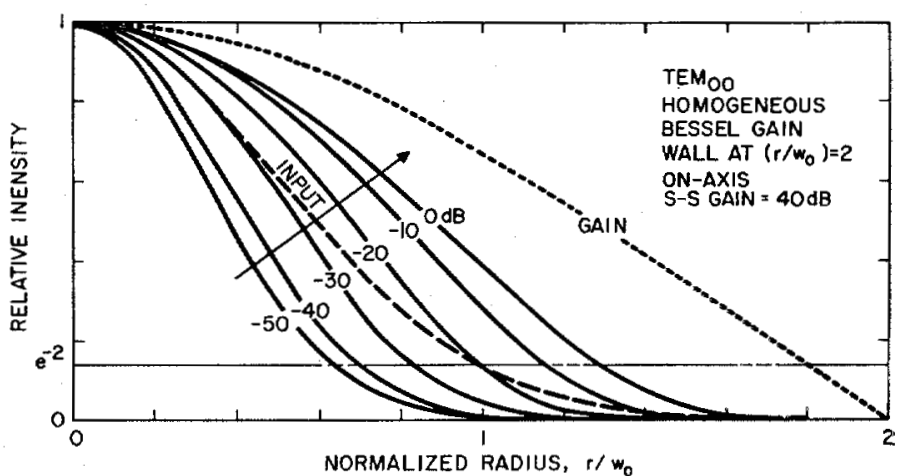

(a)

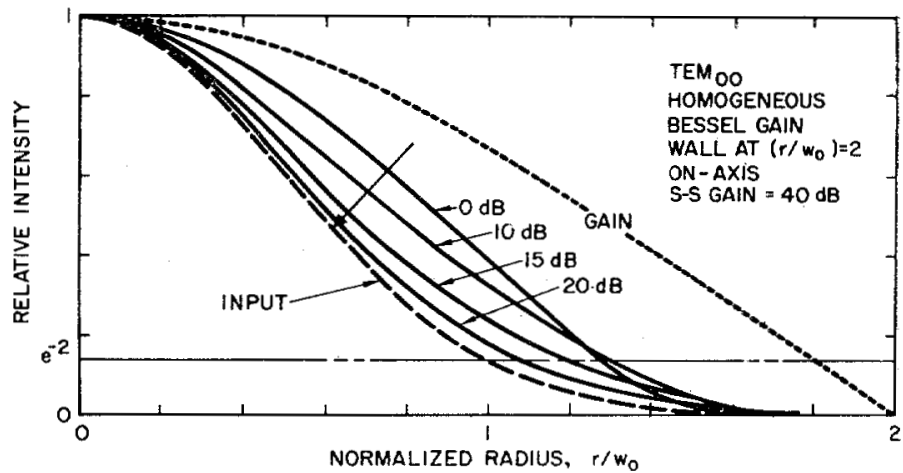

(b)

Fig. 8. Spreading of a TEM ${ }_{00}$ input beam (shown dashed) when the small-signal gain has a Bessel distribution across the tube (shown dotted, with the first zero or "wall" at $r / w_{0}=2$ ). (a) shows the beam narrowing that occurs at small input signals due to the gain distribution, followed by beam spreading as the signal level is increased from $-50 \mathrm{~dB}$ to $0 \mathrm{~dB}$; (b) shows the contraction back to the input shape as the input signal is increased from $0 \mathrm{~dB}$ to $20 \mathrm{~dB}$.

of the discrepancies in the measurement of $S_{o}$ to lack of full signal transmission.

To make matters worse, consider the curves shown in Fig. 7, which compare the four possible combinations of measurement method and type of interaction. If the type of interaction is not really known (and most gas lasers seem to fall in the "in between" category of being approximately inhomogeneous at low signal levels but approaching homogeneous interaction at high signal levels), it is more difficult to determine $S_{c}$ accurately from such a plot.

Fig. 8 shows the beam distortion under the same conditions as Fig. 2, but with a small-signal gain that decreases with radius as

$$
g_{o}(r)=g_{\circ}(0) J_{o}\left(\frac{2.405}{2} \frac{r}{w_{o}}\right)
$$

which places the tube "wall" at $r / w_{0}=2$. The $J_{\circ}$ variation is shown in Fig. 8 as the dotted curve labeled "gain". Such a variation is appropriate for diffusion-dominated gain processes, such as those commonly found in gas lasers. The results are not particularly sensitive to the form of the gain variation, however, and other distributions that fall to zero at the wall would give similar results. The same input Gaussian is shown as the dashed curve, with intensity $e^{-2}$ at $r / w_{o}=1$. We now note that 
BRIDGES: GAUSSIAN BEAM DISTORTION

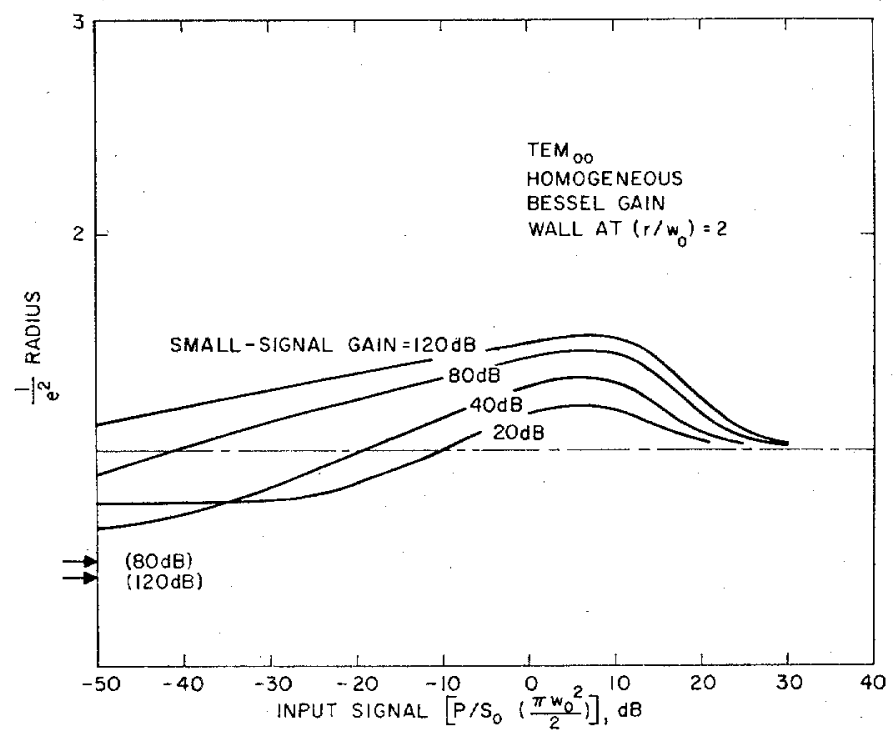

Fig. 9. Variation of the $e^{-2}$ radius with input signal intensity for different values of on-axis small-signal gain when the gain has a Bessel distribution across the tube. Asymptotic values for the 80- $\mathrm{dB}$ and $120-\mathrm{dB}$ curves are shown by arrows.

for small values of input signal, the output beam is narrowed, as we would expect from combining (7) with (13)

$$
S_{\text {out }}(r)=S_{\text {in }}(r) \exp \left[g_{\circ}(0) l J_{o}\left(\frac{2.405}{2} \frac{r}{w_{o}}\right)\right]
$$

which gives the limiting shape of the output distribution in the case of small-signal amplification. From (14) we can see that for larger $g_{0} l, S_{\text {out }}(r)$ will be narrower. As the signal increases, the beam broadens as before [Fig. $8(a)$ ] up to input signals of the order of $0 \mathrm{~dB}$. Further increase in the input signal [Fig. 8(b)] results in an ultimate approach to the input intensity distribution, as would be predicted by the asymptotic solution (9) which shows that $g_{0}$ plays an ever-decreasing role as $S_{\text {in }}$ increases. Fig. 9 summarizes the results for variation of the $e^{-2}$ radius with input signal intensity for small-signal on-axis gain of 20 to $120 \mathrm{~dB}$. The asymptotic values for smallsignal inputs were actually computed, but they could also have been obtained by solving (14) for the $e^{-2}$ radius $r_{s}$, which yields the implicit expression

$$
g_{o} l=2 \frac{1-\left(\frac{r_{e}}{w_{o}}\right)^{2}}{1-J_{o}\left(\frac{2.405}{2} \cdot \frac{r_{e}}{w_{o}}\right)} .
$$

The computed asymptotic values for $80-\mathrm{dB}$ and $120-\mathrm{dB}$ small-signal gains are indicated by arrows in Fig. 9.

Fig. 10 shows the saturation-induced distortion for the TEM $_{01}^{*}$ ("doughnut") mode. A uniform small-signal gain distribution is assumed again. Figure 10(a) shows the increasing width with increasing input up to $\sim 0 \mathrm{~dB}$. (The distribution is normalized to $r / w_{0}=0.7$, which was the closest computed radius to that producing the theoretical maximum at $r / w_{0}=0.707$, since the on-axis intensity is identically zero.) One interesting feature of this mode is that the central dark spot begins to "fill in". Further increase in power [Fig. 10(b)] shows a return

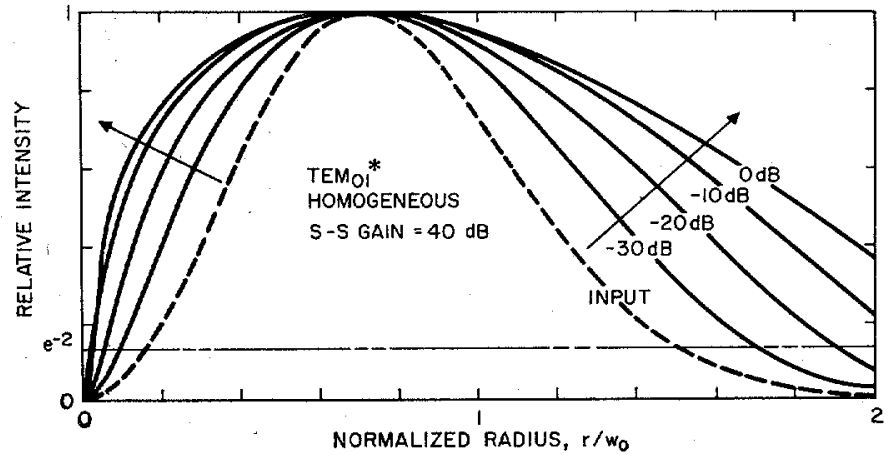

(a)

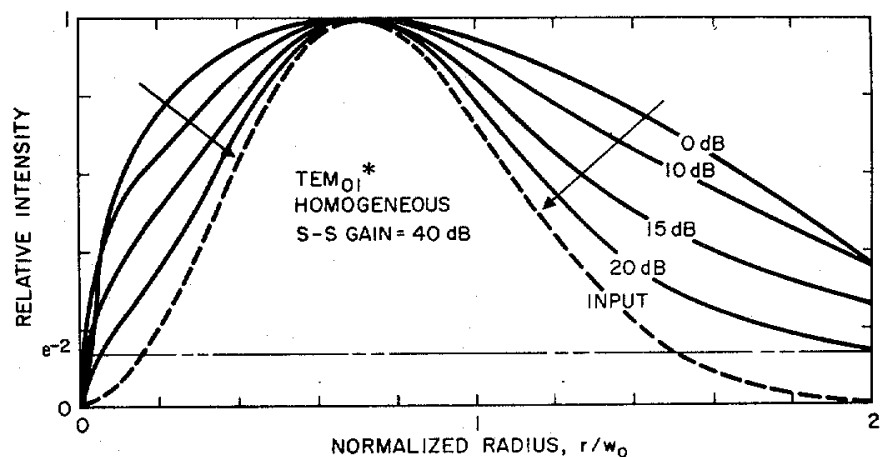

(b)

Fig. 10. Distortion of a $\mathrm{TEM}_{01}{ }^{*}$ input beam (shown dashed) for homogeneous interaction with a uniform small-signal gain of $40 \mathrm{~dB}$. (a) shows the spreading and "filling in" of the central dark dot with increasing input signals from $-30 \mathrm{~dB}$ to $0 \mathrm{~dB}$; (b) shows the shrinkage back to the input shape with signals from $0 \mathrm{~dB}$ to $20 \mathrm{~dB}$. The intensity is normalized approximately at the maximum, $r / w_{o}=0.707$.

toward the input distribution. Note that the central dark spot does not recover as fast as the gross features return to the Gaussian-Laguerre distribution. Fig. 11 repeats the same sequence for the $\mathrm{TEM}_{10}$ ("bull's-eye") mode. The bright ring increases greatly up to around $0 \mathrm{~dB}$ and then decreases as shown in Fig. 11(b). Note that the dark ring at $r / w_{0}=0.707$ fills in rapidly with increasing signal, and remains more filled-in even when the gross features have returned to their original values.

\section{Extension to Oscillators}

It is tempting to speculate on the results of calculations similar to those described above, but carried out for a laser oscillator. One might expect a change in the $\mathrm{TEM}_{\mathrm{ou}}$ mode size depending on the ratio of the intracavity flux to the saturation flux, with the passive TEM $_{00}$ mode distribution occurring for $\bar{S}_{\text {circulating }} \gg S_{o}$ and $\bar{S}_{\text {circulating }}$ $\ll S_{o}$, and a larger distorted mode for $\bar{S}_{\text {circulating }} \approx S_{o}$. (Of course we would expect this only for a uniform smallsignal gain distribution; the work of Casperson and Yariv has already confirmed the change in mode size when a radial gain distribution is present even when $S \ll S_{\circ}$.) The amplifier results given in this paper are not directly applicable because a given portion of laser medium is traversed by the beam only once, rather than several times as in an oscillator. Furthermore, the beam is assumed to be completely unapertured by the amplifier medium. Previous calculations of the Fox-Li type for 


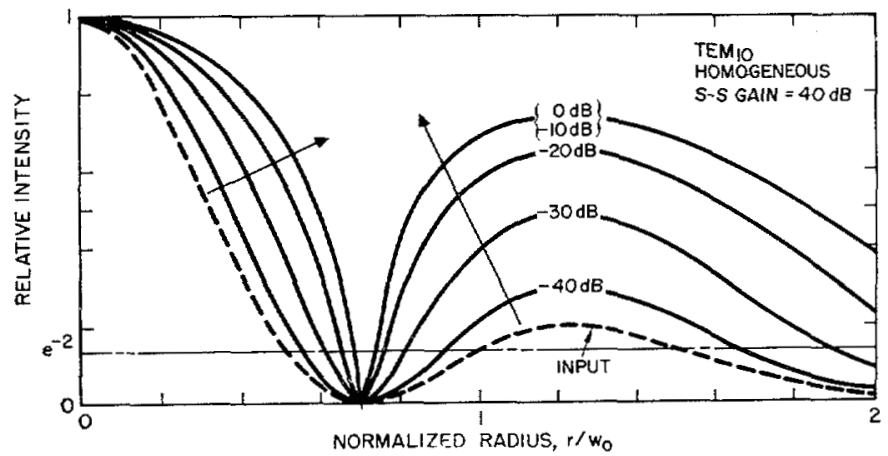

(a)

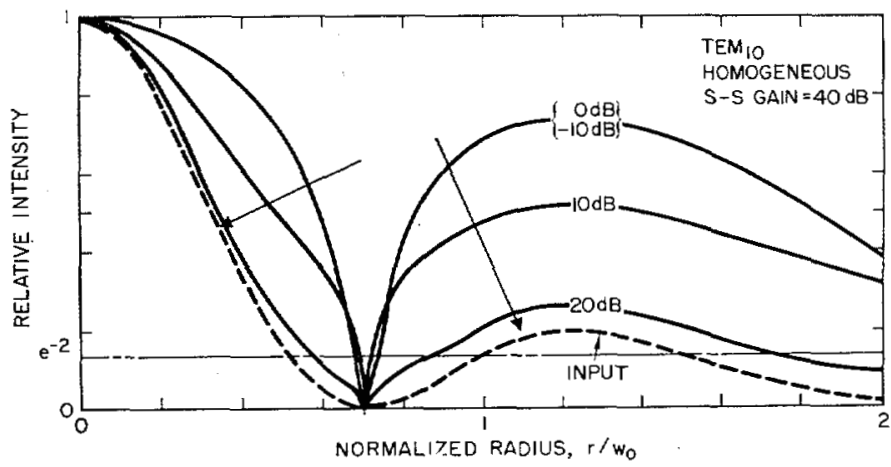

(b)

Fig. 11. Distortion of a TEM 10 input beam (shown dashed) for homogeneous interaction with a uniform small-signal gain of 40 $\mathrm{dB}$. (a) shows the spreading and "filling in" of the central dark dot with increasing imput signals from $-30 \mathrm{~dB}$ to $0 \mathrm{~dB}$; (b) shows the shrinkage back to the input shape with signals from $0 \mathrm{~dB}$ to $20 \mathrm{~dB}$. The intensity is normalized to the on-axis value.

saturable media in oscillators [17]-[19] have shown only very small departures from the passive cavity modes. However, these calculations were performed with diffraction properly included and for cavities with finite Fresnel number (that is, cavities with definite transverse boundaries). For this case, the mode size and distribution are determined primarily by the diffraction losses introduced by the aperture of the laser oscillator (mirror size), and a Gaussian mode would be the expected solution even for saturable or distorted media. (Such a mode occurs even in a laser oscillator with a highly nonuniform medium, e.g., a homogeneous medium with a small aperture midway between the mirrors; a Gaussian mode results with a size determined by that aperture rather than the mirror size.) One might argue that Gaussian-like modes will always occur in an oscillator because it is the property of these modes to "continuously reproduce themselves" on transmission (or repeated reflection). However, it may prove worthwhile to include the concepts from the model presented here in a Fox- $\mathrm{Li}$ type calculation but use values of small-signal gain and signal/saturation flux ratios of the order of those used here rather than the smaller values used in [17]-[19]. If a significant contribution is made by saturation in such an oscillator calculation, including diffraction, the saturable medium should be treated in a distributed manner as in the present work, rather than concentrated in a thin slab as in [17]-[19]. The extreme mathematical difficulty of such a treatment was of course recognized in [17]-[19], and this was the reason the simplified "slab" medium model was used.

Some further impetus is given to earrying out such a calculation by the experimental observation that the higher order modes in some gas laser oscillators do not have the simple Gaussian-Laguerre distribution. For example, the $\mathrm{TEM}_{0 \mathrm{i}}$ mode oscillating in a several-watt argon-ion laser appears to have an intensity distribution much closer to the " $0 \mathrm{~dB}$ " curve of Fig. 10 than the dotted "input" curve describing the passive TEM ${ }_{01}^{*}$ cavity mode [20]. While still other phenomena may be invoked to explain these observations, they nevertheless require explanation.

\section{Conclusions}

The simple model of a saturable laser amplifier treated in this paper has shown that significant changes in beam size and intensity distribution can result for large smallsignal gains when the input flux density is the order of the saturation flux. Saturable gain causes a general expansion of the beam, while saturable loss results in a contraction of the beam. For much stronger or weaker input signals the amount of distortion becomes small. A radial distribution of the small-signal gain that falls off away from the beam axis (such as the Bessel function $J_{o}$ ) results in less distortion for the TEM $\mathrm{TH}_{00}$ mode compared with a uniform gain distribution. The effect of beam distortion on the measurement techniques usually used to determine $S_{o}$ was discussed. Sample results for the distortion of the higher order $\mathrm{TEM}_{01}^{*}$ and $\mathrm{TEM}_{10}$ modes were given, and these results appear to be consistent with observations of these modes in some gas laser oscillator types, especially the argon-ion laser.

\section{APPENDIX}

A sample listing of one form of the GE $265 \mathrm{BASIC}$ program used in computing the results given in this paper is shown (Fig. 12). This program calculates the normalized output distribution, true power gain, on-axis gain, $3 \mathrm{~dB}$ radius, $e^{-2}$ radius, 10 percent radius, and the radii within which 50 percent and 90 percent of the output is contained.

Line 170 requires the specification of the small-signal gain in decibels. Lines 190 to 195 control the range of normalized input power for which the computation is made. Line 310 specifies the number of increments in the forward integration. Lines 320 and 330 control the range of radii covered, the maximum radius being $M^{*} \mathrm{R} 1$. Line 430 specifies the input signal distribution (and, of course, the title line 120). Line 620 specifies the radial gain distribution (uniform in this case). Lines 720 and 745 must be modified for the inhomogeneously broadened case (as well as the title line 140). The case of loss is most easily treated by making the following changes:

160 PRINT "LOSS(DB)";

$720 \operatorname{LET} \mathrm{T}(\mathrm{N})=\mathrm{Q}(\mathrm{N})^{*}(\mathrm{G}(\mathrm{M})) /\left(\left(\mathrm{EXP}\left(\mathrm{G}\left(\mathrm{M}^{*}\right) \mathrm{N}\right) /\right.\right.$ $\mathrm{Q}(\mathrm{N}))+1))$

$745 \mathrm{LET} \mathrm{T}(\mathrm{N})=\mathrm{Q}(\mathrm{N})^{*}(\mathrm{G}(\mathrm{M})) /((\operatorname{EXP}(\mathrm{G}(\mathrm{M}) * \mathrm{~N}) /$ $\mathrm{Q}(\mathrm{N}))+1))$

$755 \operatorname{LET} \phi(\mathrm{M})=\mathrm{Q}(\mathrm{N} 1)^{*} \operatorname{EXP}(-\mathrm{G}(\mathrm{M}) * \mathrm{~N} 1)$ 


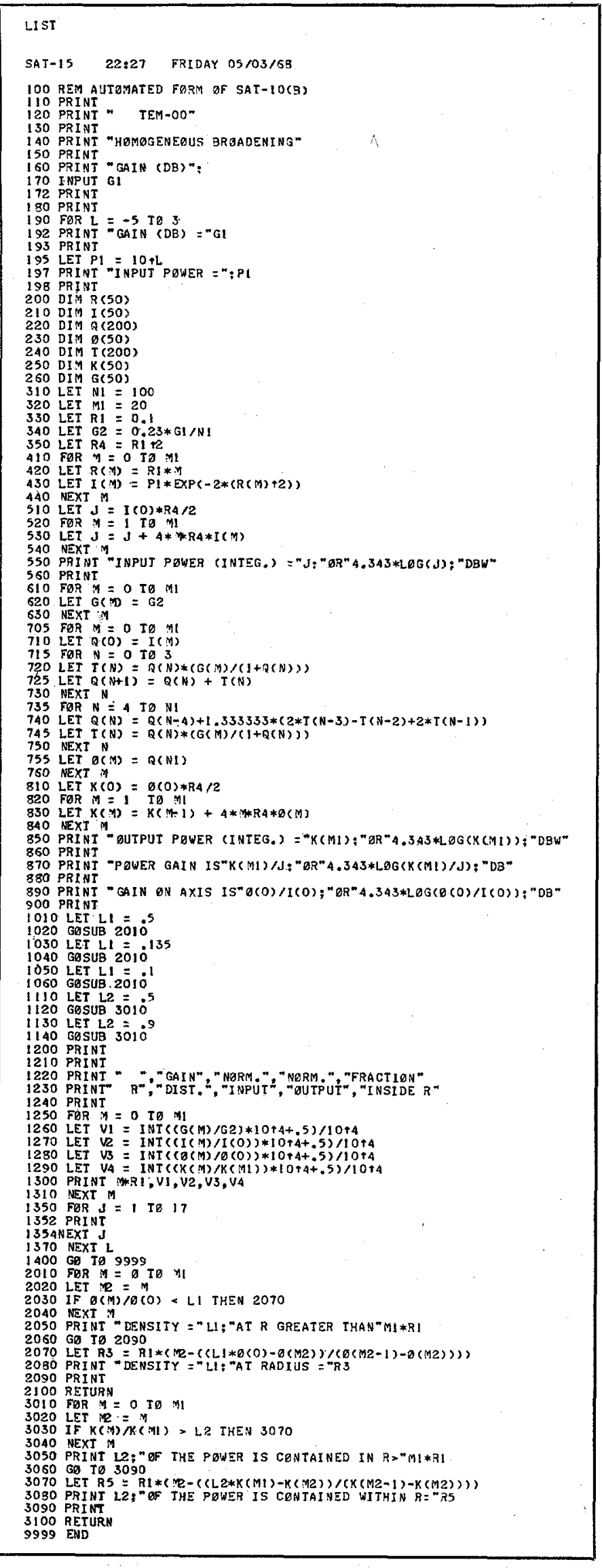

Fig. 12. and input $60-\mathrm{dB}$ loss as "60," not " -60 ," etc. The reader may satisfy himself that these are the proper equations to use when the substitution given by (10) is made in (1).

\section{ACKNOWLEDGMENT}

The author would like to acknowledge the suggestions and contributions made in several discussions of this work at Hughes Research Laboratories with his colleagues, P. O. Clark, R. J. Freiberg, D. C. Forster, A. S. Halsted, G. N. Mercer, and M. R. Smith, and also with H. Statz and A. Yariv. Credit is also due to C. R. Buckey of HRL for expediting the author's conversations with the GE 265 computer.

\section{REFERENCES}

[1] A. G. Fox and T. Li, "Resonant modes in a maser interferometer," Bell. Sys. Tech. J., vol. 40, pp. 453-488, March 1961.

[2] For a derivation of (1) and (2), see, for example, E. I. Gordon, A. D. White, and J. D. Rigden, "Gain saturation at 3.39 microns in the He-Ne laser," in Proc. Symp. on Optical Masers. New York: Polytechnic Inst. Brooklyn, April 16-19, 1963.

[3] See, for example, W. Kaplan, Ordinary Differential Equations. Reading, Mass.: Addison-Wesley, 1958, ch. 10.

[4] For the formulas relating to the Gaussian modes, see, for example, the review paper by $\mathrm{H}$. Kogelnik and $\mathrm{T}$. $\mathrm{Li}$, "Laser beams and resonators," Proc. IEEE, vol. 54, pp. 1312-1329, October 1966.

[5] This effect has been described for a laser amplifier some time ago and evaluated independently by several people, including the author. However, the first presentation the author can recall was by $R$. Kompfner at the Conference on Electron Device Research, Salt Lake City, Utah, June 1963. [7] gives the actual details of the calculation for a quadratic gain variation.

[6] That is, effect 2) combined with the "hole burning" described by W. R. Bennett, Jr., "Hole-burning effects in a He-Ne optical maser," Phys. Rev., vol. 126, pp. 580-593, April 15, 1962.

[7] H. Kogelnik, "On the propagation of Gaussian beams of light through lenslike media including those with a loss or gain variation," A ppl. Opt., vol. 4, pp. 1562-1569, December 1965.

[8] L. Casperson and A. Yariv, "The Gaussian mode in optical resonators with a radial gain profile." Appl. Phys. Letters, vol. 12, pp. 355-357, May 15, 1968.

[9] For an extensive bibliography of nonlinear effects, see, e.g., the review article by $\mathrm{N}$. Bloembergen, "The stimulated Raman effect," Am. J. Phys., vol. 35, pp. 989-1023, November 1967.

[10] See, for example, J. R. Whinnery, D. T. Miller, and F. Dabby, "Thermal convection and spherical aberration distortion of laser beams in low-loss liquids," IEEE J. Quantum Electronics (Correspondence), vol. QE-3 pp. 382-383, September 1967. This is not the original paper on the subject, but it contains the most complete bibliography on the effect.

[11] P. A. Miles and J. W. Lotus, "J-1-A high-power $\mathrm{CO}_{2}$ laser radar transmitter," presented at the 1968 International Conference on Quantum Electronics, Miami, Fla., May 14-17, 1968.

[12] M. R. Smith and D. C. Forster, "J-2-High-power laser amplifier at $10.6 \mu \mathrm{m}$," presented at the 1968 International Conference on Quantum Electronics, Miami, Fla., May 14-17, 1968.

[13] R. Carbone, private communication.

[14] A. L. Mikaeliane, V. G. Savelyev, and J. G. Turkov, "On the propagation of a ruby laser high-coherent radiation through an optical amplifier," Radiotechn. i Electron. (to be published, 1968, an English preprint was kindly forwarded to the author by Dr. Turkov).

[15] H. Kogelnik and T. J. Bridges, "A nonresonant multipass $\mathrm{CO}_{2-}$ laser amplifier," IEEE J. Quantum Electronics (Correspondence), vol. QE-3, pp. 95-96, February 1967.

[16] D. F. Hotz and J. W. Austin, "Gain saturation flux and stimulated emission cross section for the $10.6 \mu$ line of $\mathrm{CO}_{2}, "$ " Appl. Phys. Letters, vol. 11, pp. 60-62, July 15, 1967. See also Errata, vol. 11, p. 141, August 15, 1967.

[17] H. Statz and C. L. Tang, "Problem of mode deformation in optical masers," J. Appl. Phys., vol. 36, pp. 1816-1819, June 1965.

[18] A. G. Fox and T. Li, "Effect of gain saturation on the oscillating modes of optical masers," IEEE J. Quantum Electronics, vol. QE-2, pp. 774-783, December 1966.

[19] C. L. Tang and H. Statz, "Effects of intensity-dependent anomalous dispersion on the mode shapes of Fabry-Perot oscillators," J. Appl. Phys., vol. 38, pp. 886-887, February 1967.

[20] R. J. Freiberg and A. S. Halsted, "Properties of low order transverse modes in argon ion lasers," Appl. Opt. (to be published). 